\title{
IR QSOs at low and high redshifts
}

\author{
X. Y. Xia ${ }^{1,2}$, C. N. $\mathrm{Hao}^{2}$, S. D. $\mathrm{Mao}^{3,1}$, H. Wu ${ }^{2}$, and Z. G. Deng ${ }^{4,2}$ \\ ${ }^{1}$ Dept. of Physics, Tianjin Normal University, 300074 Tianjin, China. \\ email: xyxia@bao.ac.cn \\ ${ }^{2}$ National Astronomical Observatories, Chinese Academy of Sciences, \\ A20 Datun Road, 100012 Beijing, China \\ ${ }^{3}$ Univ. of Manchester, Jodrell Bank Observatory, Macclesfield, Cheshire SK11 9DL, UK \\ ${ }^{4}$ College of Physical Science, Graduate School of the Chinese Academy of Sciences, \\ 100039 Beijing, China
}

\begin{abstract}
By investigating properties from the infrared (IR) to the optical of IR-selected QSOs (IR QSOs), optically selected QSOs (PG QSOs), and narrow-line Seyfert 1 galaxies (NLS1s) in the local Universe, we find that the IR excess in IR QSOs is mostly in the far-IR, and their IR spectral indices suggest that the excess emission is caused by starbursts rather than AGNs. The ratio of the star formation rate and the accretion rate is about several hundred for IR QSOs, but decreases with the central black hole mass. We also study an optically selected QSO sample at high redshift with hyper-luminous far-IR luminosities. We find that similar to IR QSOs at low redshift, these high redshift QSOs have a far-IR excess also, which should be due to the contribution of starbursts heating the dust. But the ratio of star formation rate to accretion rate for QSOs at high redshift is typically smaller than that for IR QSOs at low redshift, which hints at the relatively faster growth of black holes at early epochs.
\end{abstract}

\section{Introduction}

In the last few years, it has become increasingly clear that the evolution of supermassive black holes and that of their host galaxies must be correlated, as there are tight correlations between the black hole mass, galactic velocity dispersion (e.g. Ferrarese \& Merritt 2000), and the mass or luminosity of the hot stellar component of the host galaxy (e.g. Kormendy \& Gebhardt 2001; Magorrian et al. 1998; Laor 1998). However, it is unclear how the correlations arise.

By studying a large sample of narrow emission line galaxies from the Sloan Digital Sky Survey (SDSS), Heckman et al. (2004) found that the volume averaged ratio of the star formation rate to the accretion rate onto the central black holes is about 1,000 for bulge-dominated galaxies. This value is in good agreement with the ratio of the bulge mass to the black hole mass derived empirically (Marconi \& Hunt 2003). However, their results are based on 23,000 narrow emission-line galaxies which excludes type 1 AGNs. It would be more intriguing to study the connection of star formation and accretion processes to the central black hole in IR-selected type 1 AGNs, as the star formation and AGN activity are coeval in these objects.

In this paper, we explore the properties of IR QSOs by comparing their properties with those of low redshift optically-selected type 1 AGNs. We also apply these diagnostics to high redshift optically-selected QSOs with hyper-luminous FIR luminosities, to shed some light on the FIR properties and starbursts-AGN connection at high redshift. Throughout this paper we adopt a cosmology with a matter density parameter $\Omega_{\mathrm{m}}=0.3$, a cosmological constant $\Omega_{\Lambda}=0.7$, and a Hubble constant of $H_{0}=70 \mathrm{~km} \mathrm{~s}^{-1} \mathrm{Mpc}^{-1}$. 


\section{Sample selection}

The aim of our study is to understand the connection between star formation and accretion processes onto central black holes. For this purpose, we used an infrared-selected type 1 AGN sample. For comparison, we also compiled an optically-selected QSO (PG QSO) sample and an NLS1 sample, for which the infrared information is available.

An optically-selected QSO sample at high redshift with millimetre observations (Carilli et al. 2001; Omont et al. 2001, 2003) was compiled also.

\section{Estimation of physical parameters}

\subsection{Black hole masses}

We estimated black hole masses by applying the virial theorem to the line-emitting gas,

$$
M_{\mathrm{BH}}=\frac{R_{\mathrm{BLR}} V^{2}}{G}
$$

where $R_{\mathrm{BLR}}$ and $V$ are the radius and velocity of the broad emission line region (BLR), respectively. For low redshift sources, the size of the BLR $\left(R_{\mathrm{BLR}}\right)$ can be estimated from the empirical relationship between the size and the monochromatic continuum luminosity at $5100 \AA$. The velocity $V$ can be estimated from the Full Width at Half Maximum (FWHM) of the $\mathrm{H} \beta$ broad emission line.

For high redshift QSOs, the black hole masses were measured directly from Vestergaard (2004) and converted to the cosmology we adopted.

\subsection{Monochromatic luminosities at $60 \mu \mathrm{m}$}

For low redshift sources, the monochromatic luminosities at $60 \mu \mathrm{m}$ were estimated directly from $\lambda L_{\lambda}=\nu L_{\nu}$. While for high redshift QSOs, we estimated the monochromatic luminosity at $60 \mu \mathrm{m}$ from the flux density at $1.2 \mathrm{~mm}$ by assuming the rest-frame FIR SED can be described by a grey-body spectrum with a dust temperature of $41 \mathrm{~K}$ and a dust emissivity of 1.95, according to Priddey \& McMahon (2001). Notice that the best-fit parameters from Priddey \& McMahon (2001) are the results without knowledge concerning the power sources heating the dust.

\subsection{Star formation rates}

The star formation rate was calculated by the monochromatic luminosity at $60 \mu \mathrm{m}$ due to starbursts. Note that the $60 \mu \mathrm{m}$ flux densities were used for this purpose because they have the fewest upper limits.

Combining the relations among the IR luminosities integrated over different wavelength coverage (Lawrence et al. 1989; Cardiel et al. 2003) and the prescription of SFR given by Kennicutt (1998), we have

$$
\mathrm{SFR} \approx 6.52 M_{\odot} \mathrm{yr}^{-1} \frac{L_{60 \mu \mathrm{m}}}{10^{10} L_{\odot}} .
$$

\section{Results}

As shown in Fig. 1a the featureless optical continuum luminosity at $5100 \AA$ correlates tightly with the broad $\mathrm{H} \beta$ luminosity for all three samples as a whole. According to Veilleux, Kim \& Sanders (1999), this relation suggests that central AGNs power the optical emission for all the sample objects. While from Fig. 1b, one can see that a considerable fraction of IR QSOs deviate from the regression line for PG QSOs and 


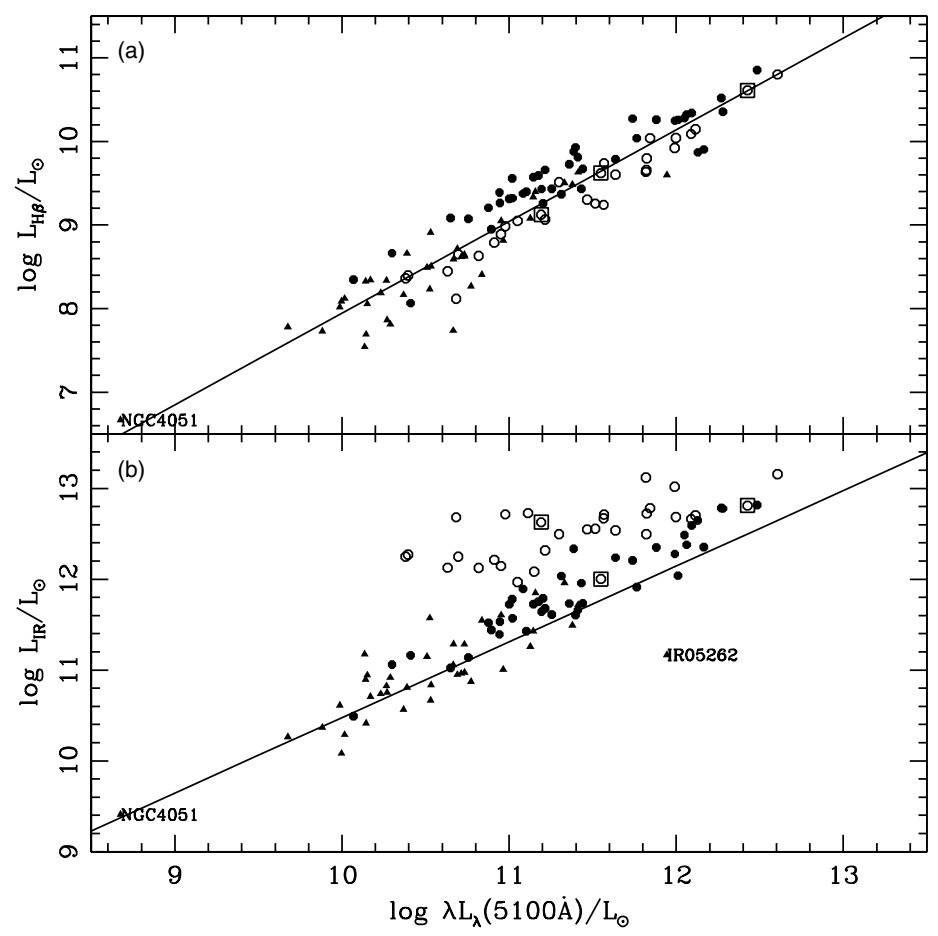

Figure 1. (a) $\mathrm{H} \beta$ luminosity (b) $L_{\mathrm{IR}}$ vs. $\lambda L_{\lambda}(5100 \AA)$. The open circles represent IR QSOs, while the filled circles and triangles represent PG QSOs and NLS1s respectively. The open circles enclosed by open squares are the three IR QSOs that are also PG QSOs. In panel (a), the solid line represents the linear regression for all IR QSOs, PG QSOs, and NLS1s, excluding NGC4051. Note that in all of our statistical analysis, NGC4051 is excluded because it is far from others in terms of all physical parameters. In panel (b), the solid line represents the linear regression for all PG QSOs and NLS1s. For clarity, upper limits of $L_{\mathrm{IR}}$ are not labelled, but survival analyses were performed for these upper limits.

NLS1s. Specifically, the IR excess of IR QSOs occurs mainly in the FIR band as shown in Fig. 2. We argue that the IR excess, especially the FIR excess of IR QSOs is due to the existence of an extra energy source to heat the dust in IR QSOs.

It is believed that $\alpha(60,25)$ is a measure of the dust temperature (e.g. Sekiguchi 1987). The larger the value, the higher the dust temperature. As shown in Fig. 3, IR QSOs have lower $\alpha(60,25)$ values and hence lower dust temperatures compared with optical AGNs statistically. This suggests that the IR excess of IR QSOs is due to star formation. Starbursts play an important role in heating the dust in IR QSOs.

IR QSOs are accreting material and forming stars at the same time. Therefore, it is an ideal laboratory for exploring the connection between black hole accretion and star formation. We find that the ratio of the star formation rate and the accretion rate is typically about several hundred for IR QSOs, which agrees with the ratio of the bulge mass to the black hole mass empirically derived (Marconi \& Hunt 2003). This agreement is consistent with the idea that the growth of central super-massive black holes and bulges are simultaneous.

The QSOs at high redshift are above the regression line for PG QSOs and NLS1s as shown in Fig. 4. If we assume that the properties of dust torii are similar in high redshift 


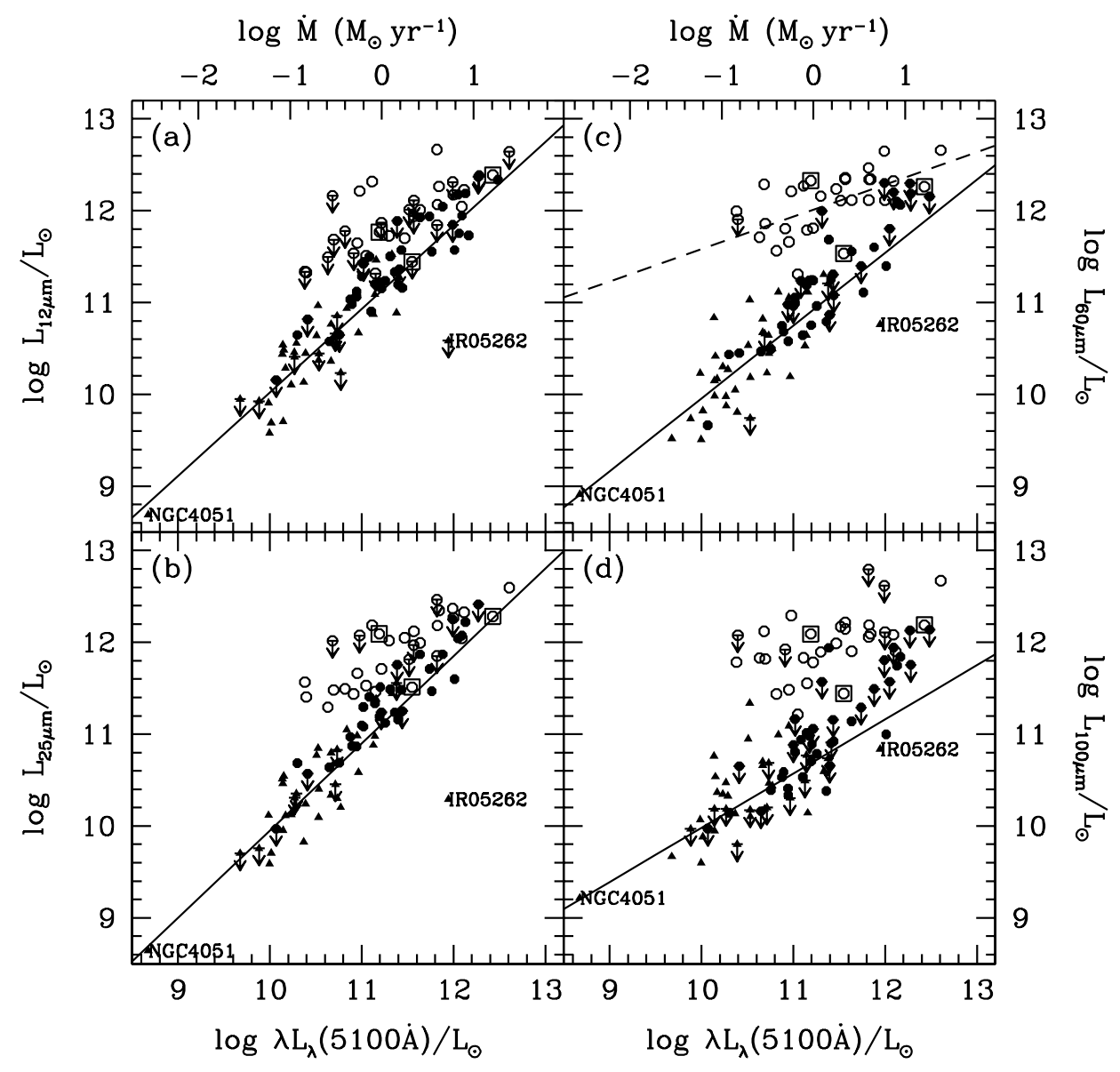

Figure 2. The monochromatic luminosities at $12 \mu \mathrm{m}, 25 \mu \mathrm{m}, 60 \mu \mathrm{m}$, and $100 \mu \mathrm{m}$ vs. $\lambda L_{\lambda}(5100 \AA)$. The symbols are the same as in Fig. 1 and the arrows denote upper limits. The solid line represents the linear regression for all the PG QSOs with available infrared flux densities and NLS1s. The accretion rate is labelled at the top abscissa. In panel (c) the best-fit line for IR QSOs is plotted as the dashed line.

QSOs to those at low redshift, the QSOs at high redshift have a FIR excess also. The extrapolation of the properties of IR QSOs at low redshift results in the conclusion that star formation is important for heating the dust in the QSOs at high redshift.

Fig. 5 shows the ratio of the star formation rate to the accretion rate onto the central black hole versus the black hole mass for IR QSOs and 11 QSOs at redshifts of about 4 from Carilli et al. (2001). Unfortunately, the trend of high redshift QSOs in this figure depends on the prescription of SFR, specifically the assumption of an IMF. However, in spite of the uncertainty of the SFR estimation, we can still see that the ratio of SFR to accretion rate for QSOs at high redshift is typically smaller than that for IR QSOs at low redshift, which hints at a faster growth of black holes at early epochs.

\section{Acknowledgements}

This project is supported by the NSF of China, under grant numbers 10333060 , 10273012 , and TG1999075404. 


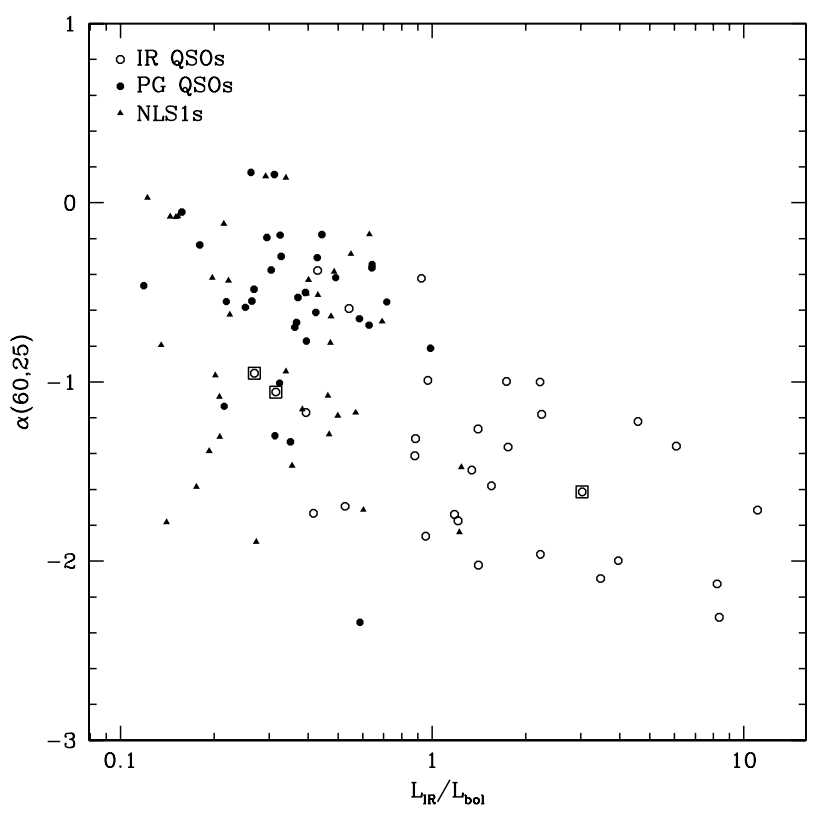

Figure 3. The infrared spectral index, $\alpha(60,25)$, versus the infrared excess, $L_{\mathrm{IR}} / L_{\mathrm{bol}}$. The symbols are the same as in Fig. 1.

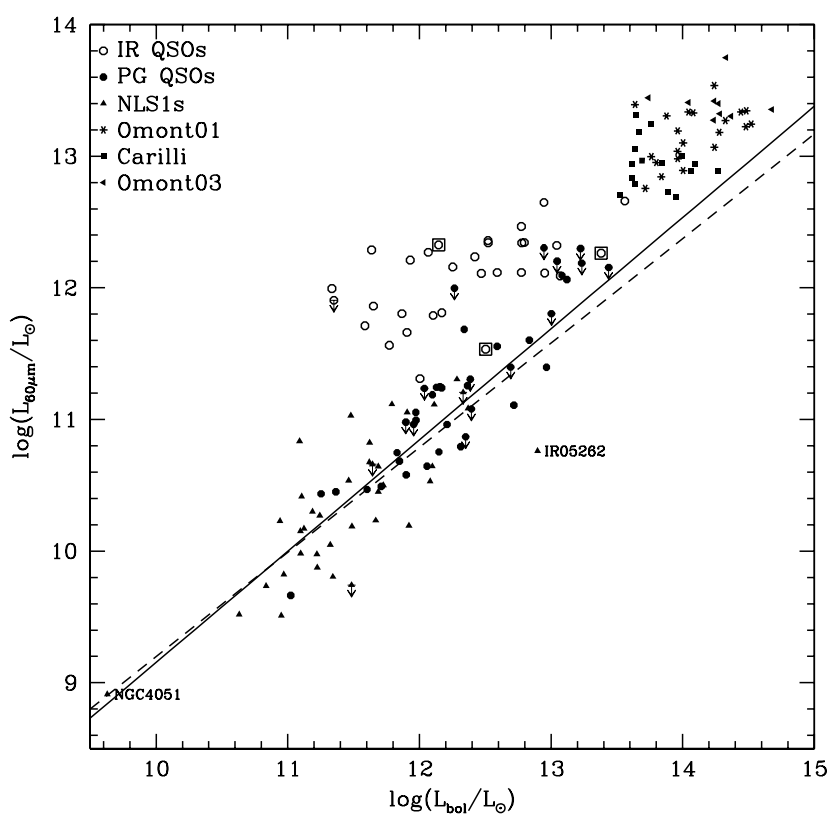

Figure 4. The monochromatic luminosity at $60 \mu \mathrm{m}$ vs. the bolometric luminosity of AGN. The symbols for objects at low redshift are the same as those in Fig. 1. The stars and squares represent the QSOs at redshifts of about 4, while the rotated triangles represent the QSOs at redshifts of about 2. The dashed line is the best-fit line for all PG QSOs and NLS1s using survival analysis. The solid line is the best-fit line for all PG QSOs and NLS1s with detected flux densities at $60 \mu \mathrm{m}$. 


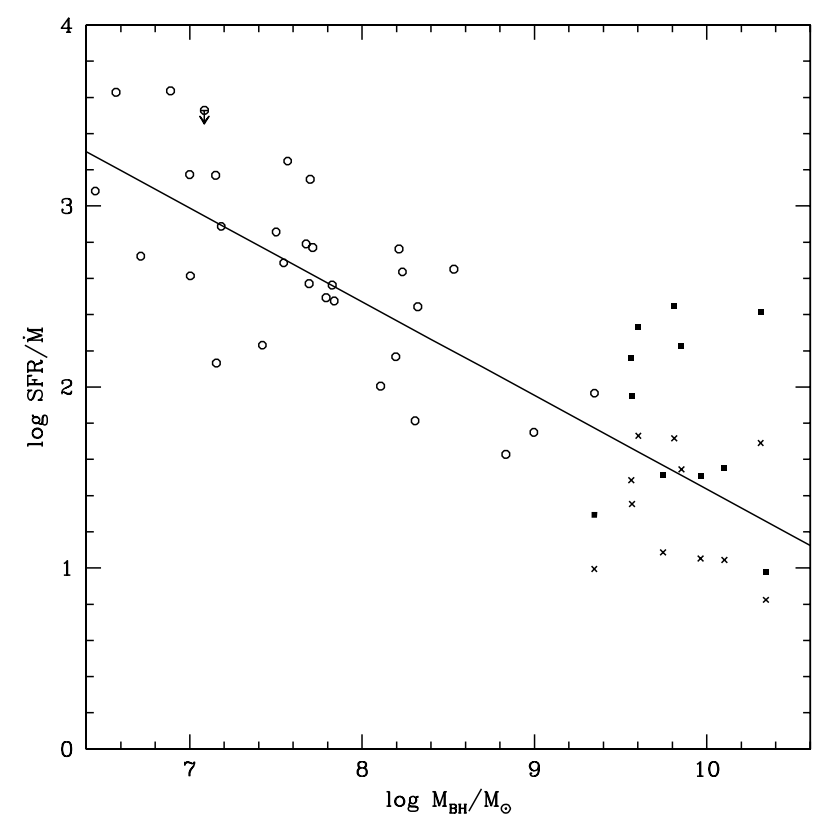

Figure 5. The ratio of the star formation rate to the accretion rate vs. the black hole mass. The open circles represent IR QSOs at low redshift, while the crosses and squares represent the QSOs at redshift of about 4. The squares denote the SFR calculated by the formula applied to IR QSOs at low redshift, while the crosses indicate that a flatter IMF was assumed according to Omont et al. (2001). The black hole masses of QSOs at high redshift were measured from Fig 7 in Vestergaard (2004). The solid line is the best regression line for IR QSOs at low redshift.

\section{References}

Cardiel, N., Elbaz, D., Schiavon, R. P., Willmer, C. N. A., Koo, D. C., Phillips, A. C., Gallego, J., 2003, ApJ, 584, 76

Carilli, C. L., et al., 2001, ApJ, 555, 625

Ferrarese, L., Merritt, D., 2000, ApJ, 539, L9

Heckman, T. M., Kauffmann, G., Brinchmann, J., Charlot, S., Tremonti, C., White, S. D. M., 2004, ApJ, 613, 109

Kennicutt, R. C., 1998, ARA\&A, 36, 189

Kormendy, J., Gebhardt, K., 2001, in The 20th Texas Symposium on Relativistic Astrophysics, ed. H. Martel \& J. C. Wheeler, AIP (astro-ph/0105230)

Laor, A., 1998, ApJ, 505, L83

Lawrence, A., Rowan-Robinson, M., Leech, K., Jones, D. H. P., Wall, J. V., 1989, MNRAS, 240, 329

Magorrian, J., et al., 1998, AJ, 115, 2285

Marconi, A., Hunt, L. K., 2003, ApJ, 589, L21

Omont, A., Beelen, A., Bertoldi, F., Cox, P., Carilli, C. L., Priddey, R. S., McMahon, R. G., Isaak, K. G., 2003, A\&A, 398, 857

Omont, A., Cox, P., Bertoldi, F., McMahon, R. G., Carilli, C., Isaak, K. G., 2001, A\&A, 374, 371

Priddey, R. S., McMahon, R. G., 2001, MNRAS, 324, L17

Sekiguchi, K., 1987, ApJ, 316, 145

Veilleux, S., Kim, D.-C., Sanders, D. B., 1999, ApJ, 522, 113

Vestergaard, M., 2004, ApJ, 601, 676 